\title{
Support-Vector-Machine-Based Reduced-Order Model for Limit Cycle Oscillation Prediction of Nonlinear Aeroelastic System
}

\author{
Gang Chen, ${ }^{1}$ Yingtao Zuo, ${ }^{2}$ Jian Sun, ${ }^{1}$ and Yueming $\mathrm{Li}^{1}$ \\ ${ }^{1}$ State Key Laboratory for Strength and Vibration of Mechanical Structures, School of Aerospace, \\ $X i^{\prime}$ an Jiaotong University, Xi'an, Shannxi 710049, China \\ ${ }^{2}$ School of Aeronuatics, Northwestern Polytechnical University, Xi'an, Shannxi 710072, China
}

Correspondence should be addressed to Yingtao Zuo, zuoyingtao@nwpu.edu.cn

Received 25 June 2011; Accepted 3 November 2011

Academic Editor: Hamdy Nabih Agiza

Copyright (C) 2012 Gang Chen et al. This is an open access article distributed under the Creative Commons Attribution License, which permits unrestricted use, distribution, and reproduction in any medium, provided the original work is properly cited.

\begin{abstract}
It is not easy for the system identification-based reduced-order model (ROM) and even eigenmode based reduced-order model to predict the limit cycle oscillation generated by the nonlinear unsteady aerodynamics. Most of these traditional ROMs are sensitive to the flow parameter variation. In order to deal with this problem, a support vector machine- (SVM-) based ROM was investigated and the general construction framework was proposed. The two-DOF aeroelastic system for the NACA 64A010 airfoil in transonic flow was then demonstrated for the new SVMbased ROM. The simulation results show that the new ROM can capture the LCO behavior of the nonlinear aeroelastic system with good accuracy and high efficiency. The robustness and computational efficiency of the SVM-based ROM would provide a promising tool for real-time flight simulation including nonlinear aeroelastic effects.
\end{abstract}

\section{Introduction}

Aeroelasticity is the science concerned with the fluid-structure interaction including the inertial, elastic, and aerodynamic forces. The prediction of aeroelastic instability in the transonic regime plays a very important role in the definition of the flight envelope for many highperformance aircraft. For example, flutter and limit cycle oscillation (LCO) are the major nonlinear aeroelastic unstable phenomena, which are very dangerous to the aircraft structure. With the development of computational aeroelasticity, the nonlinear aeroelastic response can be accurately predicted by the high-fidelity physics-based CFD/CSD couple solver. However, the use of multistep time domain calculations for each aircraft state is computationally expensive and provides limited insight into the dependence of the parameters on the type 
of response in the vicinity of the instability boundary. In order to reduce the expensive computational cost, a novel conception called reduced-order model (ROM) based on highfidelity physics model has been put forward in recent years. ROM seeks to capture the dominant nonlinear behavior of the aeroelastic system by constructing a simple mathematical representative model, which is very convenient to be used in conceptual design, control, and data-driven systems [1].

Different approaches for reduced-order modeling of aerodynamic systems have been investigated, including linearization about a nonlinear steady-state flow data-driven model such as Volterra theory of nonlinear systems [2] and linear model fitting ARMA model [3], representation of the aerodynamic system in terms of its eigenmodes such as POD method $[4,5]$, and representation of the nonlinear aerodynamic system using the nonlinear dynamic theory [6]. Many approaches for constructing linear flow and aeroelastic ROMs have been developed and shown to produce good numerical results that compare well with high-fidelity nonlinear solvers. Most aeroelastic phenomena such as flutter and gust response can be deal with these ROMs based on the dynamically linearized equation. However, unfortunately, some important strong nonlinear dynamic phenomena such as LCO cannot be simulated by the small disturbance solvers. For modeling the cases where the amplitude of the flow perturbation is large, the ROMs based dynamically nonlinear solvers are required. Thomas et al. proposed a new nonlinear HB/ROM which can predict the LCO of the NLR 7301 airfoil aeroelastic model very well in the transonic regime [7]. Badcock et al. put forward a fully nonlinear ROM construction method based on bifurcation theory, which can predict LCO very well [8]. Recently, we also proposed a new dynamically nonlinear NPOD/ROM, which enables the rapid modeling of nonlinear unsteady flows for the prediction and control of LCO $[9,10]$. These recently developed high-order ROMs for LCO simulation are much more complex than traditional ROMs and are also not easy to realize in code.

Nonlinear system-identification-based ROMs have been widely used to predict the transonic flutter boundary such as Volterra series $[11,12]$ and neural network approaches [13, 14]. Because of the high efficiency and simplicity, it is an attractive idea to predict the LCO by system-identification-based ROMs. Neural networks have been widely applied in nonlinear system identification because of the ability of self-learning, strong parallel processing, and fault tolerance $[15,16]$. There are also successful applications in the LCO prediction of the airfoil aeroelastic model [17, 18]. However, the disadvantages of getting stuck into local minima, over-fitting, and low generalization performance prevent the practical application of these methods. Recently, support vector machine introduced by Vapnik [19] has become a promising tool for solving nonlinear regression problems including nonlinear system identification. SVM is a newly emerging technique for learning relationships in data within the framework of statistical learning theory and structural risk minimization. Relying on statistical learning theory which enables learning machines to generalize well to unseen data, SVM has been significantly highlighted in the areas of system identification and parameter estimation [20,21]. In comparison with neural networks, SVM has strict theory and mathematical foundation. It does not have the problem of local optimization and dimensional disaster and can achieve higher generalization performance for small samples.

In this study, we develop an SVM-based ROM for predicting the LCO induced by the nonlinear aerodynamics with high efficiency and good accuracy. Firstly, we gave a brief introduction about the regression SVM machine; secondly, we proposed a general construction framework of the SVM-based ROM for the aeroelastic system; finally, we demonstrated the ROM by the two-DOF NACA 64A010 airfoil aeroelastic model in detail. 


\section{Support Vector Machine-Based Nonlinear System Identification}

The basic idea of SVM theory is to map the data to a higher-dimensional feature space via nonlinear mapping functions and then do the linear regression in this space. SVM-based nonlinear system identification approaches have taken advantage of both the kernel trick and the well-developed SVR algorithmic implementations [19, 22]. After introducing $\varepsilon$-insensitive loss function, SVMs extend to the regression case, named support vector regression (SVR), whose key idea is to replace regression line with a $\varepsilon$-tube. Geometrically, the samples located outside this tube will not be considered in regression model. The goal of SVR is to find a decision function $f(x)$ which has at most $\varepsilon$ deviation from actually obtained observations and at the same time is as flat as possible. Therefore, in the process of minimizing the empirical errors, SVRs also try to maximize the generalization ability.

Based on statistical learning theory, SVRs can be applicable especially to small-sample learning problems. Here, we give a brief summary of SVR. Given an input-output data set $\left\{\left(x_{1}, y_{1}\right), \ldots,\left(x_{l}, y_{l}\right)\right\} \subset R^{d} \times R$, such as the time response series of the unsteady aerodynamic coefficient of the aeroelastic system, SVR can be formulated as [19]

$$
\begin{aligned}
& \min _{\mathbf{w}, b, \xi_{,}, \xi^{*}} \frac{1}{2}\|\mathbf{w}\|^{2}+C \sum_{i=1}^{l}\left(\xi_{i}+\xi_{i}^{*}\right) \\
& \text { subject to }\left\{\begin{array}{l}
y_{i}-\left\langle\mathbf{w}, \Phi\left(x_{i}\right)\right\rangle-b \leq \varepsilon+\xi_{i} \\
\left\langle\mathbf{w}, \Phi\left(x_{i}\right)\right\rangle+b-y_{i} \leq \varepsilon+\xi_{i}^{*} \\
\xi_{i}, \xi_{i}^{*} \geq 0, \quad i=1,2, \ldots, l
\end{array}\right.
\end{aligned}
$$

where the $\mathbf{w}$ is the weighting vector and $\varepsilon$-insensitive loss function is defined as

$$
L\left(y_{i}, f\left(x_{i}\right)\right)= \begin{cases}0, & \text { if }\left|y_{i}-f\left(x_{i}\right)\right| \leq \varepsilon \\ \left|y_{i}-f\left(x_{i}\right)\right|-\varepsilon, & \text { otherwise }\end{cases}
$$

The slack variables $\xi_{i}, \xi_{i}^{*}$ determine the sample's deviation from $\varepsilon$-tube, and the regularization parameter $C>0$ determines the trade-off between empirical risk and generalization term and punishes the samples which violate the $\varepsilon$-tube. Applying the method of Lagrange multipliers to (2.1), equation (2.1) is transformed into the following equation:

$$
\begin{aligned}
L(\mathbf{w}, b, \alpha)= & \frac{1}{2}\|\mathbf{w}\|^{2}+C \sum_{i=1}^{l}\left(\xi_{i}+\xi_{i}^{*}\right)-\sum_{i=1}^{l}\left(\eta_{i} \xi_{i}+\eta_{i}^{*} \xi_{i}^{*}\right) \\
& -\sum_{i=1}^{l} \alpha_{i}\left(\varepsilon+\xi_{i}-y_{i}+\left\langle\mathbf{w}, \Phi\left(x_{i}\right)\right\rangle+b\right) \\
& -\sum_{i=1}^{l} \alpha_{i}^{*}\left(\varepsilon+\xi_{i}^{*}+y_{i}-\left\langle\mathbf{w}, \Phi\left(x_{i}\right)\right\rangle-b\right),
\end{aligned}
$$

where $\alpha_{i}, \alpha_{i}^{*}$ are the introduced Lagrange multipliers and denoted by $\alpha_{i}^{(*)}$. 
After calculating the partial derivatives of $L(\mathbf{w}, b, \boldsymbol{\alpha})$ with respect to $\left(\mathbf{w}, b, \xi_{i}, \xi_{i}^{*}\right)$, the dual optimization problem can be obtained as follows [23]:

$$
\begin{aligned}
\min _{\alpha, \alpha^{*}} & \frac{1}{2} \sum_{i, j=1}^{l}\left(a_{i}^{*}-\alpha_{i}\right)\left(a_{j}^{*}-\alpha_{j}\right)\left\langle\Phi\left(x_{i}\right), \Phi\left(x_{j}\right)\right\rangle+\varepsilon \sum_{i=1}^{l}\left(a_{i}^{*}+\alpha_{i}\right)-\sum_{i=1}^{l} y_{i}\left(a_{i}^{*}-\alpha_{i}\right) \\
\text { s.t. } & \sum_{i=1}^{l}\left(a_{i}^{*}-\alpha_{i}\right)=0, \quad 0 \leq \alpha_{i}, a_{i}^{*} \leq C, i=1,2, \ldots, l .
\end{aligned}
$$

Equation (2.4) is a quadratic programming problem. After calculating the values of $\alpha$ and $\alpha^{*}$ for all samples, the decision function is obtained as follows:

$$
f(x)=\sum_{i=1}^{l}\left(\alpha_{i}-a_{i}^{*}\right) K\left(x_{i}, x\right)+b .
$$

In (2.5), only some samples come with nonzero $\boldsymbol{\alpha}^{(*)}$, which is called the support vector and provide sparsity. Moreover, $K\left(x_{i}, x\right)=\left\langle\Phi\left(x_{i}\right), \Phi\left(x_{j}\right)\right\rangle$ is kernel function which only depends on dot products between observations $x_{i}$ and $x_{j}$ and does not have to know nonlinear mapping $\Phi$ explicitly. Any function satisfying Mercer's condition can be used as the kernel function. The widely used kernel functions are including polynomial kernel, the Gaussian kernel, and Radial Basis kernel. From the implementation point of view, training SVM is equivalent to solving a linearly constrained quadratic programming problem with the number of variables twice that of the training data points. Many effective optimization algorithms were proposed for solving the regression problem such as the solution path algorithm [24, 25].

\section{SVM-Based Reduced-Order Model for Aeroelastic System}

\subsection{Numerical Couple Simulation Method of the Nonlinear Aeroelastic System}

The full-coupled nonlinear semi-discrete aeroelastic equation is given as

$$
\begin{gathered}
(A(u) w)_{, t}+F(w, u, v)=0, \\
M v_{, t}+f^{\text {int }}(u, v)=f^{\text {ext }}(u, w) .
\end{gathered}
$$

Equations (3.1) represent the fluid and structure dynamics, respectively, where $w$ is conservative flow field value, $F$ is flow flux residual, $A$ is flow cell volume, $u$ is the structural general displacement, $v$ is structure general displacement derivatives, $M$ is mass matrix, and $f^{\text {int }}$ is structure inner force. Also, $f^{\text {ext }}$ is the aerodynamic force acting on the structure which is dependent on the flow state values and the structure state values. Many kinds of accurate CFD/CSD couple numerical algorithms have been developed to predict the response of structure and aerodynamics simultaneously [26]. The procedure of the popular loosely couple numerical simulation algorithm is illustrated in Figure 1. A general multiblock structure mesh-based CFD/CSD loosely coupled solver was developed by the authors and 


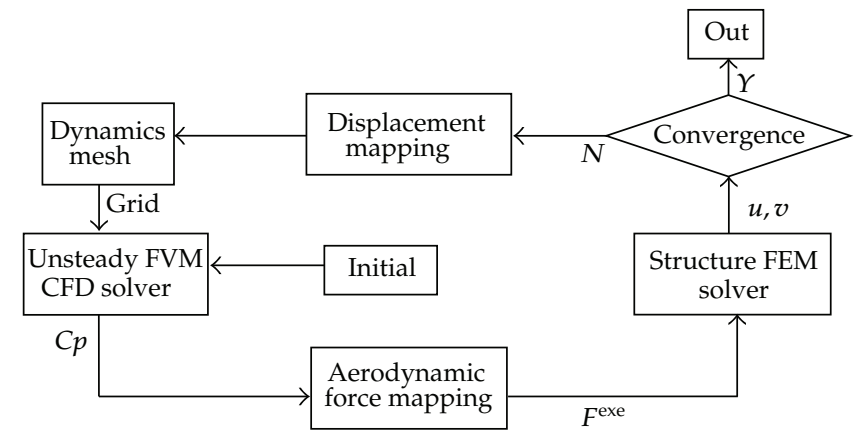

Figure 1: CFD/CSD couple algorithm.

successfully predicted the flutter and LCO for many aeroelastic models with good accuracy, including NLR 7301 airfoil section aeroelastic model, the AGARD 445.6 wing, and Goland+ wing models $[5,9,10]$.

The typical two degrees of freedom (2DOF) aeroelastic model with plunge and pitch freedom has been widely used to validate the LCO prediction method [27, 28]. As illustrated in Figure 2, defining the dimensionless time $\tau=\omega_{\alpha} t$, the aeroelastic equation can be rewritten in the nondimensional form:

$$
\left[\begin{array}{cc}
1 & x_{\alpha} \\
x_{\alpha} & r_{\alpha}^{2}
\end{array}\right]\left[\begin{array}{c}
\ddot{h} \\
\ddot{\alpha}
\end{array}\right]+\left[\begin{array}{cc}
\left(\frac{\omega_{h}}{\omega_{\alpha}}\right)^{2} & 0 \\
0 & r_{\alpha}^{2}
\end{array}\right]\left[\begin{array}{l}
\bar{h} \\
\alpha
\end{array}\right]=\frac{1}{\pi} V^{* 2}\left[\begin{array}{c}
-C_{L} \\
C_{M}
\end{array}\right],
$$

where $h$ and $\alpha$ are the airfoil plunge and airfoil pitch angle, $r_{\alpha}$ is the radius of gyration of airfoil about elastic axis, and $x_{\alpha}$ is the airfoil static dimensionless distance between the center of gravity position and the hinge axis. Also, $\omega_{h}$ and $\omega_{\alpha}$ are the uncoupled natural frequency of plunge and pitch, $\mu=m /\left(\pi \rho_{\infty} b^{2}\right)$ is the mass ratio velocity of the free flow, and $V^{*}=$ $U_{\infty} /\left(\omega_{\alpha} b \sqrt{\mu}\right)$ is the reduced velocity. In each simulation loop, after the unsteady lift $C_{L}$ and pitch moment coefficients $C_{M}$ computed by the unsteady CFD solver, the structure response $(\bar{h}, \alpha)$ can then be calculated by the multistep Runge-Kutta algorithm at the same time.

\subsection{Construction Framework of the SVM-Based ROM}

For CFD/CSD couple simulation, most of the computational cost is spent in solving the unsteady aerodynamics. The reduced-order model method is introduced for unsteady flow to improve the computational efficiency in the aeroelastic simulation. In the aerodynamic subsystem, the inputs are the structure state values $u$ and $v$, and the outputs are the generalized aerodynamic force coefficient vector $f$. The sampling time series data $(u, v, f)$ can be computed by the CFD/CSD couple solver. For example, the inputs of the 2-DOF aeroelastic system are $(\bar{h}, \alpha)$ and outputs are $C_{L}$ and $C_{M}$. In order to construct the SVMbased ROM according to (2.5) and obtain the SVR, the sampling data should be prepared carefully. Considering the time delay effect of unsteady flow, the input features are selected as $x_{i}=\left(\left(u_{i}, v_{i}\right),\left(u_{i-1}, v_{i-1}\right), \ldots,\left(u_{i-r}, v_{i-r}\right), f_{i-1}, \ldots, f_{i-s}\right)$. The $r$ and $s$ are the time delay orders dependent on the users' selection. Then, we could construct the new training sample $\left(x_{i}, f_{i}\right)$ 


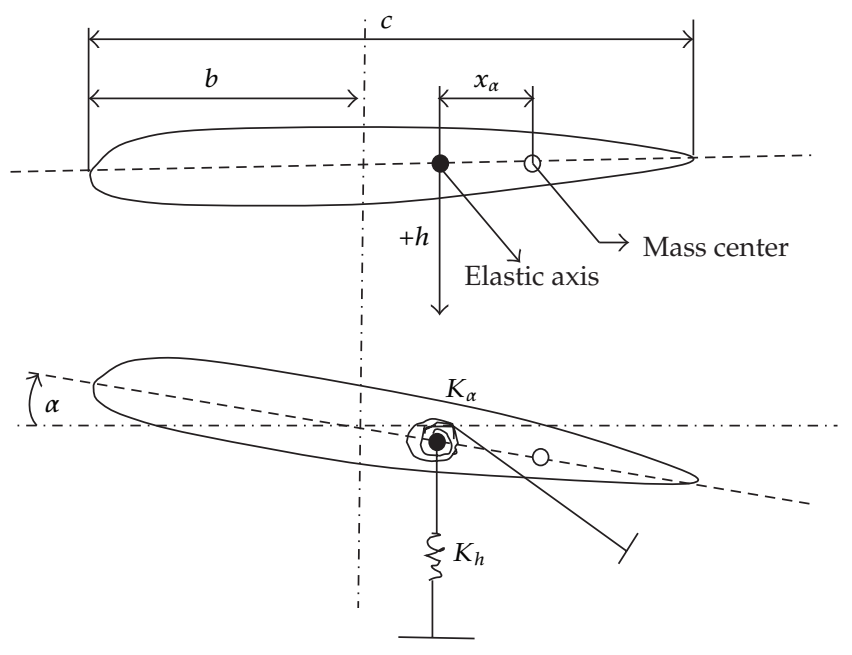

Figure 2: Two-DOF aeroelastic model.

for the SVRs from the unsteady CFD simulation results. The $\varepsilon$-path is selected as the modeling algorithm for its better accuracy and lower computational cost than other methods [24, 25].

The flow chart of the construction procedure of the SVM-based aeroelastic ROM is illustrated in Figure 3. There are five main steps in the framework. First, we construct multiple groups of input signals and compute the corresponding nonlinear aerodynamic responses by the unsteady CFD solver which is the full-order model. Here, white noise with different amplitude is selected as the training signals, because white noise can represent the natural dynamic characteristic of nonlinear system very well [27-29]. Second, the input signals and time series unsteady aerodynamics are combined to create the training samples $\left(x_{i}, f_{i}\right)$. As indicated as before, the traditional ROMs such as POD/ROM and Volterra/ROM are very sensitive to the flow parameters such as Mach number. In order to improve the robustness of the SVM-based ROM, flow parameters such as Mach number can also be treated as extra system features which can be add to the training samples, for example, $\left(\left(x_{i}, M a\right), f_{i}\right)$. Third, the SVM solution path algorithm is executed to obtain the identification model and then validate the accuracy $\left(x_{i}, f_{i}\right)$ with checking samplings. Fourth, the identification model is used to predict the unsteady aerodynamic force in applied cases. Finally, coupling the structure subsystem and aerodynamic subsystem represented by the SVM-based ROM, the aeroelastic response can be predicted according to the virtual line loop in the Figure 3 with good accuracy and high efficiency.

\section{Simulation Results and Discussions}

\subsection{Unsteady Aerodynamics Validation}

As the initial demonstration of the SVM-based ROM, the NACA 64A010 airfoil model was selected. The structure parameters are $x_{\alpha}=1.8, r_{\alpha}^{2}=3.48, a=-2.0, \mu=60.0, \omega_{h} / \omega_{\alpha}=1$. The $240 \times 120$ O-type mesh was used for the Euler solver which is the full-order model. The pitching angle is the input data, and the unsteady aerodynamic loads $C_{L}$ and $C_{M}$ are the outputs, which is an SIMO system. By federating the inputs such as the pitching and plunge to CFD solver one by one, the ROM can be used to model the MIMO system. 


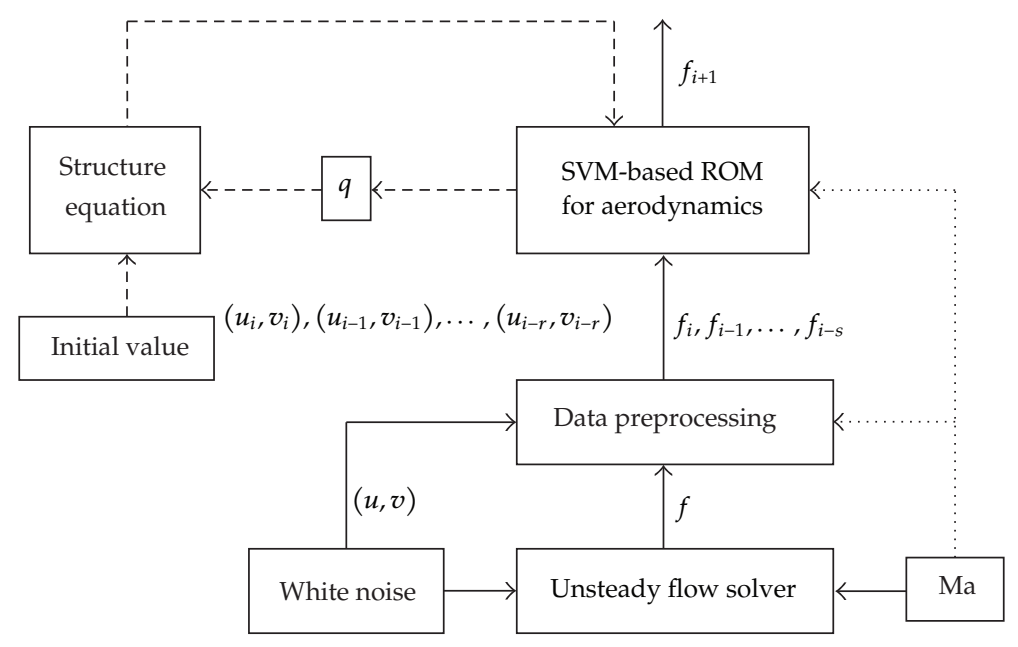

Figure 3: The workflow of SVM-based ROM.

At the given Mach number 0.825, two white noise signals for pitch motion whose amplitudes are 0.01 and 0.2 , respectively, were selected and federated into the CFD solver to compute the time series response of the unsteady aerodynamic coefficients. Selecting $r=3$, $s=4$, and preparing the training samplings for the SVR, the SVM-based ROM for unsteady aerodynamics can be constructed according to the procedure described in Section 3.2. Then, the unsteady aerodynamic loads predicted by the SVM-based ROM were compared with the CFD/Euler solver. Figure 4 plots the time responses of the lift and moment coefficients predicted by the CFD solver and the SVM-based ROM, in which the airfoil was given a forced pitch vibration movement. The results of the different models are very close, which indicated that the SVM-based ROM can predict the nonlinear aerodynamic loads with good accuracy. It took nearly 10 minutes for the CFD solver to obtain the simulation results while only about 10 seconds for the ROM in a PC. This means that SVM-based ROM has good computational efficiency for unsteady aerodynamics prediction.

\subsection{LCO Prediction}

The accuracy of the SVM-based ROM has been verified for the unsteady aerodynamics. Now, we would like to couple the SVM-based ROM with the structure dynamics equation and investigate its effectiveness in aeroelastic response analysis. At the Mach number 0.825 and reduced velocity $V^{*}=0.8$, we run the SVM-based aeroelastic model and CFD/CSD couple solver with the initial plunge velocity value 0.1 , respectively. Figure 5 shows the structural response of the simulations. The nonlinear LCO behavior of the aeroelastic model was captured by both the ROM and CFD/CSD couple solver very well. Furthermore, the predictions of the ROM agree well with the CFD/CSD couple solver.

In order to check the capability of the ROM, we construct another SVM model at Mach $=0.8$ to investigate the LCO behavior. We succeed to capture some LCO at $V^{*}=1.2$, $1.5,1.8$, and 2.0 by ROM very quickly which was shown in Figure 6 . The results had been compared with the CFD/CSD couple solver and they also agree well. Because we do not know in which condition the LCO will come out, we must use many guess initial values to 


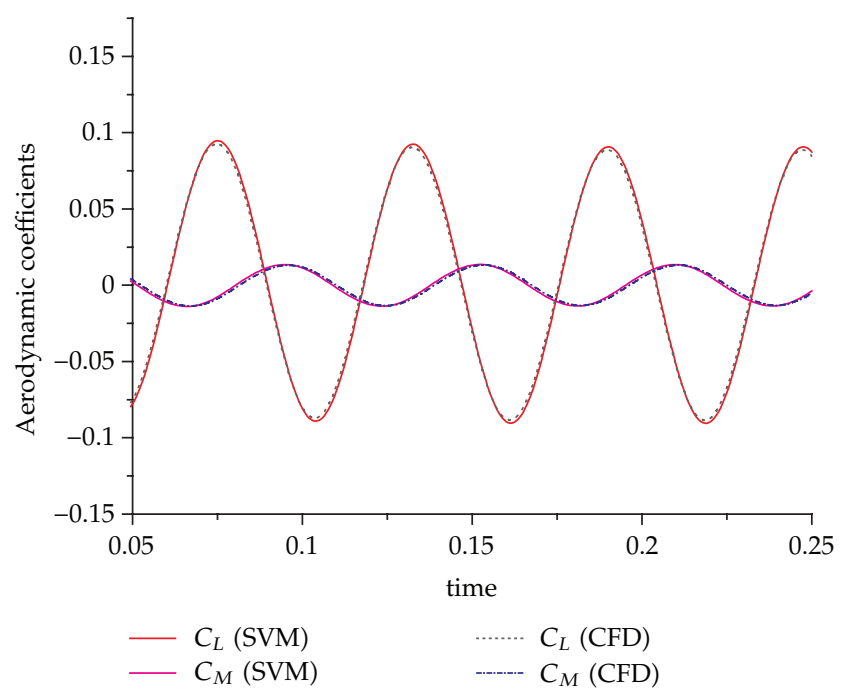

Figure 4: Comparison of aerodynamic response at Mach $=0.825$.

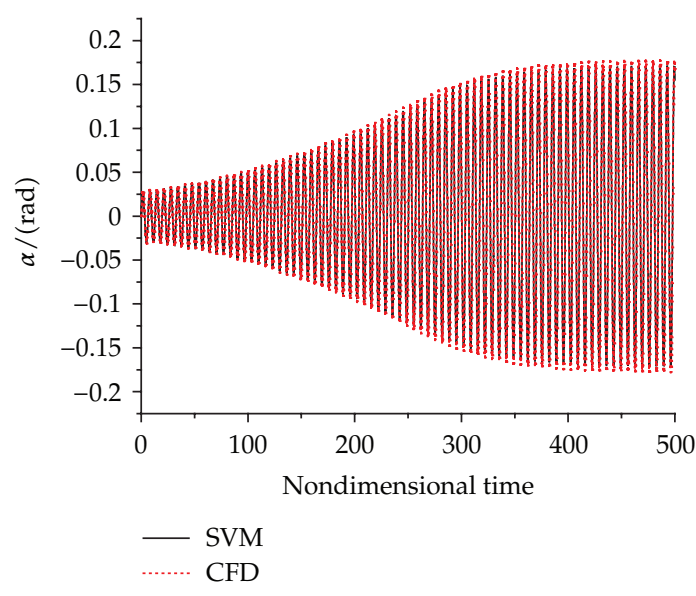

(a)

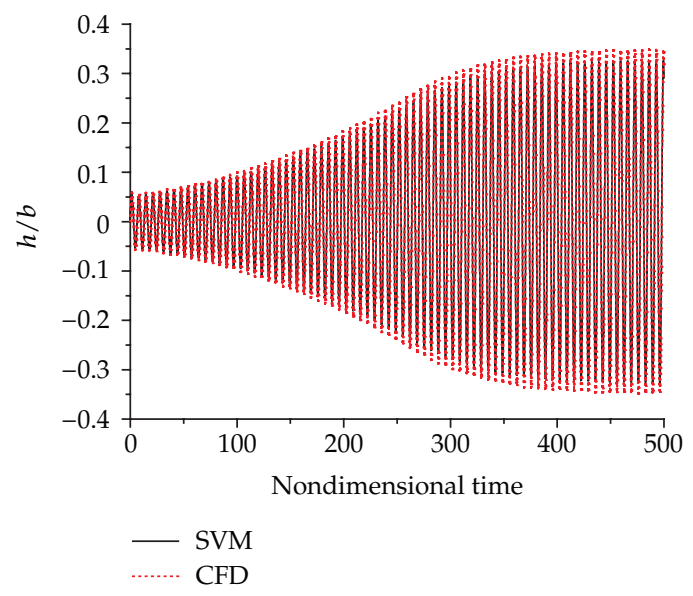

(b)

Figure 5: Comparison of LCO response at Mach $=0.825$, and $V^{*}=0.8$.

run the solver. So nearly ten hours were spent for the CFD/CSD, coupled solver to capture the LCO, while no more than ten minutes for the ROM. The high efficiency of the ROM is a very good practical merit for searching the flight envelope of the aircraft in the whole flight regime.

\subsection{Parameters Varying Simulation}

\subsubsection{ROM Construction Method}

As noted previously, the traditional ROMs such as POD/ROM and Volterra/ROM are very sensitive to the flow parameter variation, especially for the Mach numbers and Reynolds 


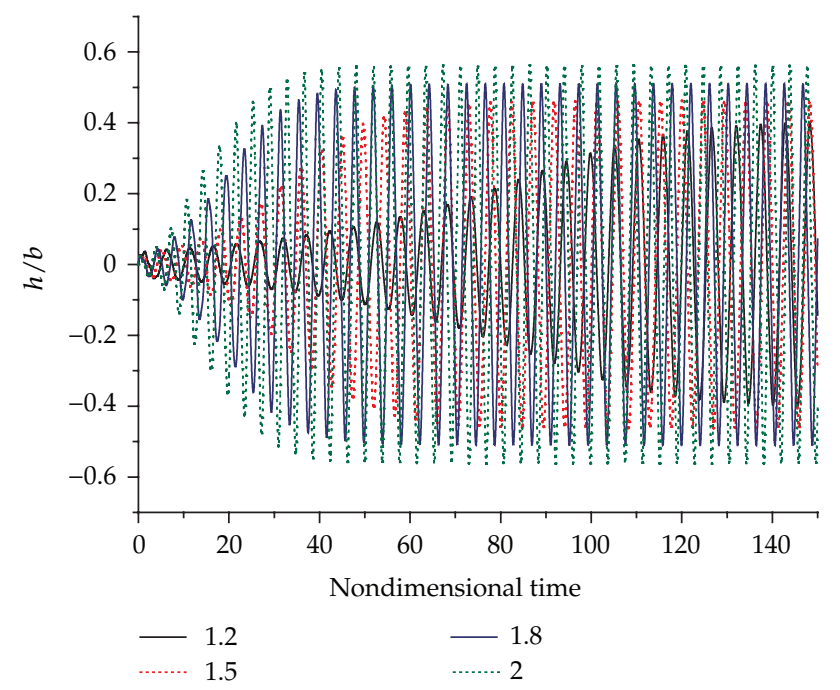

Figure 6: $\mathrm{LCO}$ response at different reduced velocity at Mach $=0.8$.

numbers [30,31]. This disadvantage prevents ROMs to be widely used in aeroelastic control, flight simulation, and some other data-driven applications [32]. Amsallem and Farhat proposed an adapting POD/ROM based on manifold interpolation which is adaptive to Mach number and the angle of attack $[30,31]$. Although their adaptive POD/ROM can predict flutter very well, no application in LCO prediction was demonstrated. Here, we demonstrate the capability of the SVM-based ROM for predicting the LCO with Mach number variation.

Firstly, we prepare the unsteady responses $C_{L}$ and $C_{M}$ at four Mach numbers such as $0.8,0.825,0.875$, and 0.8925 . Secondly, let the Mach number be another feature when we construct the training samples for SVR. This means that the structure of the training samples is $x_{i}=\left(\left(u_{i}, v_{i}\right),\left(u_{i-1}, v_{i-1}\right), \ldots,\left(u_{i-r}, v_{i-r}\right), f_{i-1}, \ldots, f_{i-s}, M a\right)$. Thirdly, the SVM-based ROM was trained and constructed according to the stand procedure as described in Section 3.2. Finally, the new SVM-based ROM can be used to predict the aeroelastic response.

\subsubsection{LCO Prediction with Varying Mach Number}

Figure 7 shows the simulation results of the SVM-based ROM and CFD/CSD couple Euler solver at Mach $=0.85, V^{*}=0.75$. The SVM-based ROM can still succeed to capture the $\mathrm{LCO}$ and also agrees well with direct couple numerical simulation of the full-order solver. These results indicate that the capability and efficiency of the SVM-based ROM to predict the aeroelastic response with parameters variation. The good robustness with the system varying parameters would provide a useful and powerful tool for near real-time elastic vehicle flight simulation. Although the difference between the ROM and CFD are a little larger than the case in Section 4.2, it is still a good and acceptable result.

In order to further validate the performance of the SVM-based ROM, we calculated the LCO behavior of the aeroelastic system in different Mach numbers. All of the reduced velocity is $V^{*}=1.2$. Figure 8 plots the LCO amplitude predicted by the SVM-based ROM and full-order CFD solver. It can be founded that in the sampling Mach numbers such as 


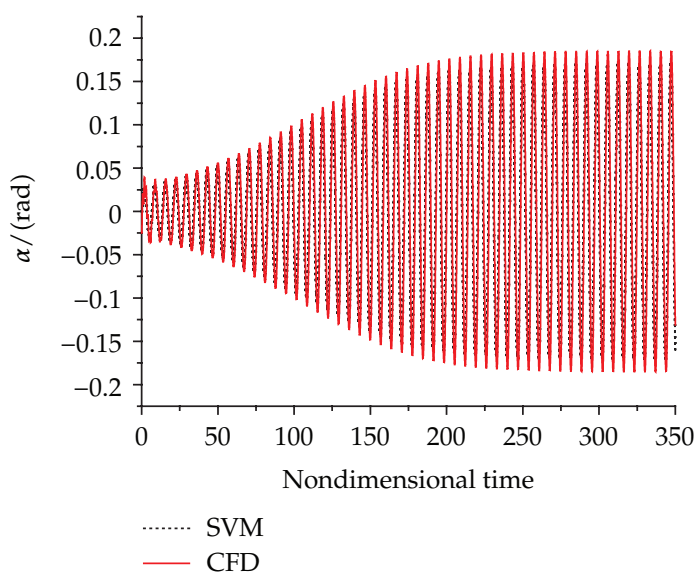

(a)

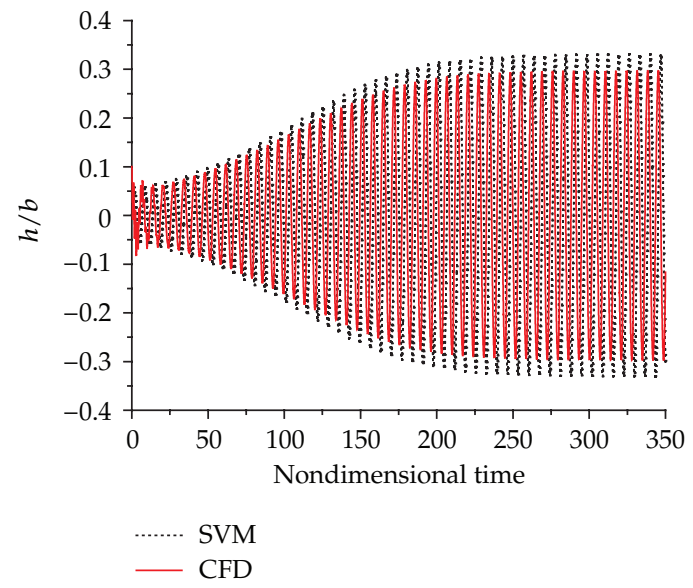

(b)

Figure 7: Comparison of LCO response at Mach $=0.85$, and $V^{*}=0.75$.

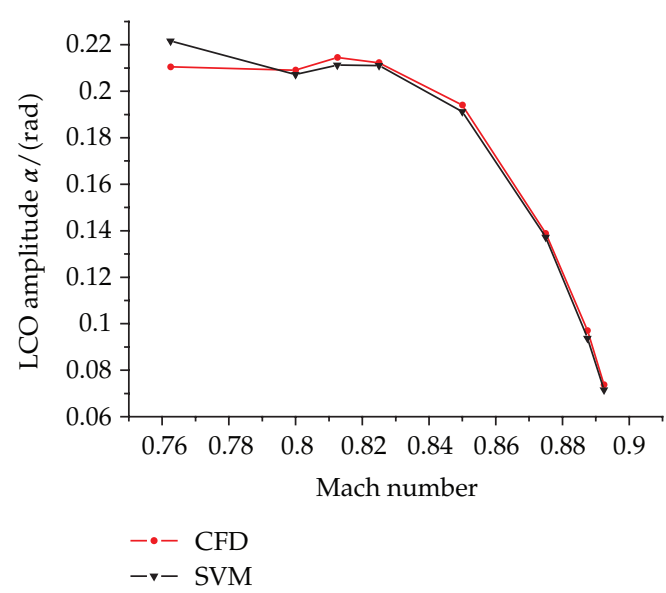

(a)

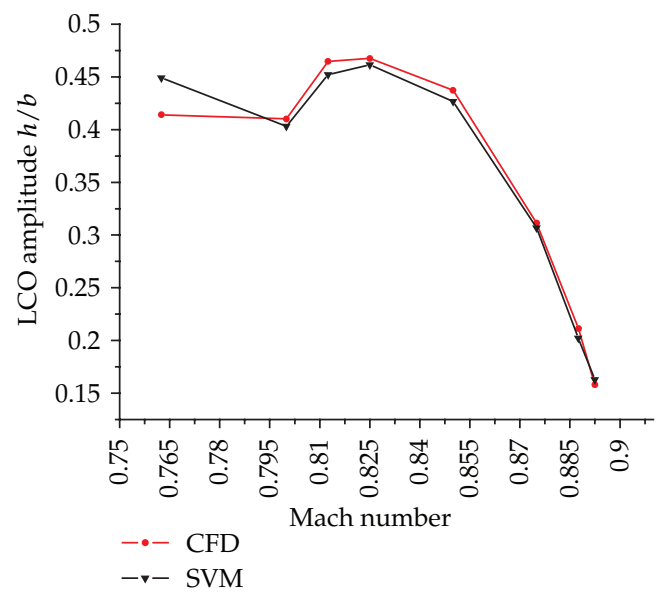

(b)

Figure 8: The LCO amplitude with Mach number (ROM versus full-order model).

$0.8,0.825,0.875$, and 0.8925 , the results predicted by SVM and CFD solver agree very well. The average error is around 5\%. This validates the conclusion of Section 4.2 again. The most important is to evaluate the accuracy of the predicted results by SVM in other Mach numbers. The difference of the results predicted by SVM and CFD is obviously and a little larger than those in the sampling Mach numbers, but it is still good enough and acceptable. Of course the obvious errors can be reduced further by adding much more samplings. This indicates that the SVM-based ROM has the capability to represent the dominant LCO response of the system with varying parameters.

There is also an interesting thing that outside of the sampling Mach number set, such as at 0.7625 , the development trend of LCO predicted by SVM does not agree with CFD solver, but the error is not so large. Maybe it is because of that SVR has excellent learning capability and generalization capability. We also investigated the LCO response above the 
Mach number 0.9, but the SVM model fail to capture the LCO. It seems that the aeroelastic system runs into divergence or convergence from the simulation of the full-order solver. It is obviously and reasonable that no data-driven model can do everything.

It takes about 1 hour for the CFD solver to capture the LCO response, but it is no more than one minutes for the SVM-based ROM to predict the same response. The computational efficiency of ROM is obvious, which is very important to the near real-time flight dynamic simulation and flight controller design. The fast prediction of the system response with good accuracy is one of the most important factors to realize these challenge applications successfully.

\section{Conclusion}

An SVM-based reduced-order model was developed for fast prediction of the response of the nonlinear aeroelastic system. We proposed a general construction framework for the SVMbased ROM. The two-dimensional aerofoil aeroelastic system was used to demonstrate the capability and performance of the ROM in detail. The simulation results show the capability, accuracy, and high efficiency of the SVM-based ROM for the LCO prediction. The SVM-based $\mathrm{ROM}$ can also fairly predict the LCO response of the 2DOF aeroelastic model with the Mach number variation. The robustness of the SVM-based ROM with varying flow parameters provides a useful tool for real-time flight simulation of flexible vehicle. Further research will focus on developing new training methods and improving the accuracy of the ROM, especially for the cases with parameters variation.

\section{Acknowledgments}

This work was supported by the National Natural Science Foundation of China (10902082, 91016008), New Faculty Research Foundation of XJTU, and the Fundamental Research Funds for the Central Universities (xjj20100126). The first author acknowledges W. T. Mao for the discussion of SVM algorithms. All the authors thank H. N. Agiza and the reviewers for their good comments.

\section{References}

[1] D. J. Lucia, P. S. Beran, and W. A. Silva, “Reduced-order modeling: new approaches for computational physics," Progress in Aerospace Sciences, vol. 40, no. 1-2, pp. 51-117, 2004.

[2] W. A. Silva and R. E. Bartels, "Development of reduced-order models for aeroelastic analysis and flutter prediction using the CFL3Dv6.0 code," Journal of Fluids and Structures, vol. 19, no. 6, pp. 729$745,2004$.

[3] J. Y. Kwak, W. Hong, S. J. Shin, I. Lee, J. W. Yim, and C. Kim, "CFD-Based aeroelastic analysis of the X-43 hypersonic flight vehicle," in Proceedings of the 39th Aerospace Sciences Meeting and Exhibit (AIAA'01), 2001.

[4] K. C. Hall, J. P. Thomas, and E. H. Dowell, "Proper orthogonal decomposition technique for transonic unsteady aerodynamic flows," AIAA Journal, vol. 38, no. 10, pp. 1853-1862, 2000.

[5] G. Chen, Y.-M. Li, G.-R. Yan, M. Xu, and X.-A. Zeng, "A fast aeroelastic response prediction method based on proper orthogonal decomposition reduced order model," Journal of Astronautics, vol. 30, no. 5, pp. 1765-1769, 2009 (Chinese).

[6] K. J. Badcock and M. A. Woodgate, "Fast prediction of transonic aeroelastic stability and limit cycles," AIAA Journal, vol. 45, no. 6, pp. 1370-1381, 2007 (Chinese).

[7] J. P. Thomas, E. H. Dowell, and K. C. Hall, "Using automatic differentiation to create a nonlinear reduced-order-model aerodynamic solver," AIAA Journal, vol. 48, no. 1, pp. 19-24, 2010. 
[8] K. J. Badcock, S. Timme, and S. Marques, "Transonic aeroelastic simulation for instability searchesand uncertainty analysis," Progress in Aerospace Sciences, vol. 47, pp. 392-423, 2011.

[9] G. Chen, Y. Li, and G. Yan, "A nonlinear POD reduced order model for limit cycle oscillation prediction," Science China, vol. 53, no. 7, pp. 1325-1332, 2010.

[10] G. Chen, Y.-M. Li, and G.-R. Yan, "Limit cycle oscillation prediction and control design method for aeroelastic system based on new nonlinear reduced order model," International Journal of Computational Methods, vol. 8, no. 1, pp. 77-90, 2011.

[11] D. E. Raveh, "Identification of computational-fluid-dynamics based unsteady aerodynamic models for aeroelastic analysis," Journal of Aircraft, vol. 41, no. 3, pp. 620-632, 2004.

[12] W. A. Silva, "Simultaneous excitation of multiple-input/multiple-output CFD-based unsteady aerodynamic systems," Journal of Aircraft, vol. 45, no. 4, pp. 1267-1274, 2008.

[13] F. D. Marques and J. Anderson, "Identification and prediction of unsteady transonic aerodynamic loads by multi-layer functionals," Journal of Fluids and Structures, vol. 15, no. 1, pp. 83-106, 2001.

[14] K. L. Lai, K. S. Won, E. P. C. Koh, and H. M. Tsai, "Flutter simulation and prediction with CFD-based reduced-order model," in Proceedings of the 47th AIAA/ASME/ASCE/AHS/ASC Structures, Structural Dynamics and Materials Conference (AIAA '06), pp. 5229-5245, Newport, RI, USA, May 2006.

[15] S. Srivastava, M. Singh, M. Hanmandlu, and A. N. Jha, "Identification of nonlinear systems using wavelets and neural network," IETE Journal of Research, vol. 52, no. 4, pp. 305-313, 2006.

[16] E. Marcelo, A. K. S. Johan, and D. M. Bart, "Kernel based partially linear models and nonlinear identification," IEEE Transactions on Automatic Control, vol. 50, no. 10, pp. 1602-1606, 2005.

[17] M. R. Johnson and C. M. Denegri, "Comparison of static and dynamic artificial neural networks for limit cycle oscillation prediction," in Proceedings of the 42nd AIAA/ASME/ASCE/AHS/ASC Structures, Structural Dynamics and Exhibit Technical Papers (AIAA '01), pp. 793-800, April 2001.

[18] O. Voitcu and Y. S. Wong, "An improved neural network model for nonlinear aeroelastic analysis," in Proceedings of the 44th AIAA/ASME/ASCE/AHS/ASC Structures, Structural Dynamics, and Materials Conference (AIAA '03), pp. 825-835, April 2003.

[19] V. N. Vapnik, "An overview of statistical learning theory," IEEE Transactions on Neural Networks, vol. 10, no. 5, pp. 988-999, 1999.

[20] I. Goethals, K. Pelckmans, J. A. K. Suykens, and B. De Moor, “Subspace identification of Hammerstein systems using least squares support vector machines," IEEE Transactions on Automatic Control, vol. 50, no. 10, pp. 1509-1519, 2005.

[21] Z. Lu and J. Sun, "Non-Mercer hybrid kernel for linear programming support vector regression in nonlinear systems identification," Applied Soft Computing Journal, vol. 9, no. 1, pp. 94-99, 2009.

[22] I. Witten and E. Frank, Data Mining Practical Machine Learning Tools and Techniques, Elsevier, 2005.

[23] A. J. Smola and B. Schölkopf, "A tutorial on support vector regression neurocolt," Tech. Rep., Royal Holloway College, London, UK, 1998.

[24] G. Wang, D. Y. Yeung, and F. H. Lochovsky, "A new solution path algorithm in support vector regression," IEEE Transactions on Neural Networks, vol. 19, no. 10, pp. 1753-1767, 2008.

[25] W. Mao, G. Yan, and L. Dong, "Weighted solution path algorithm of support vector regression based on heuristic weight-setting optimization," Neurocomputing, vol. 73, no. 1-3, pp. 495-505, 2009.

[26] R. Kamakoti and W. Shyy, "Fluid-structure interaction for aeroelastic applications," Progress in Aerospace Sciences, vol. 40, no. 8, pp. 535-558, 2004.

[27] J. P. Thomas, E. H. Dowell, and K. C. Hall, "Nonlinear inviscid aerodynamic effects on transonic divergence, flutter, and limit-cycle oscillations," AIAA Journal, vol. 40, no. 4, pp. 638-646, 2002.

[28] W. Mao, D. Hu, and G. Yan, "A new SVM regression approach for mechanical load identification," International Journal of Applied Electromagnetics and Mechanics, vol. 33, no. 3-4, pp. 1001-1008, 2010.

[29] M. Anna, B. D. P. Andrea, and J. Schoukens, "Nonlinear system identification by means of Svms: choice of excitation signals," in Proceedings of the 16th TC4 International Symposium, Exploring New Frontiers of Instrumentation and Methods for Electrical and Electronic Measurements (IMEKO '08), Florence, Italy, September 2008.

[30] D. Amsallem and C. Farhat, "Interpolation method for adapting reduced-order models and application to aeroelasticity," AIAA Journal, vol. 46, no. 7, pp. 1803-1813, 2008.

[31] D. Amsallem, J. Cortial, and C. Farhat, "Toward real-time computational-fluid-dynamics-based aeroelastic computations using a database of reduced-order information," AIAA Journal, vol. 48, no. 9, pp. 2029-2037, 2010.

[32] P. Hu, M. Bodson, and M. Brenner, "Towards real-time simulation of aeroservoelastic dynamics for a flight vehicle from subsonic to hypersonic regime," in Proceedings of the Atmospheric Flight Mechanics Conference and Exhibit (AIAA '08), Honolulu, Hawaii, USA, August 2008. 


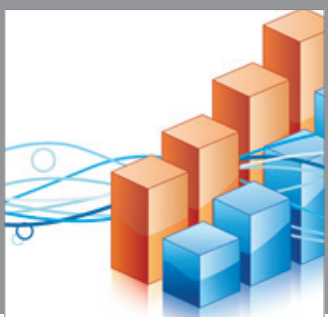

Advances in

Operations Research

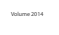

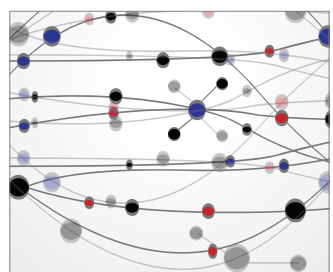

\section{The Scientific} World Journal
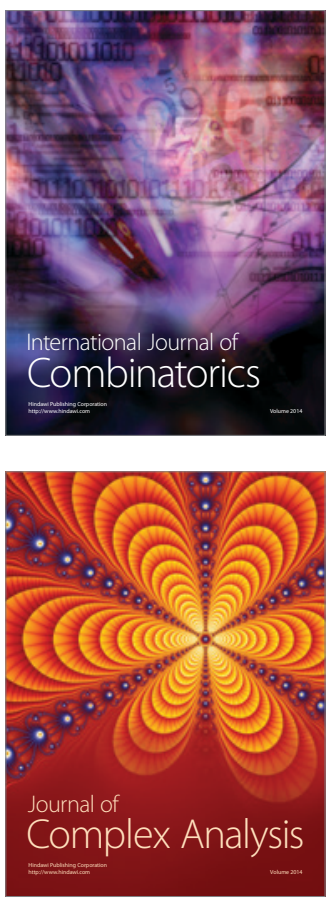

International Journal of

Mathematics and

Mathematical

Sciences
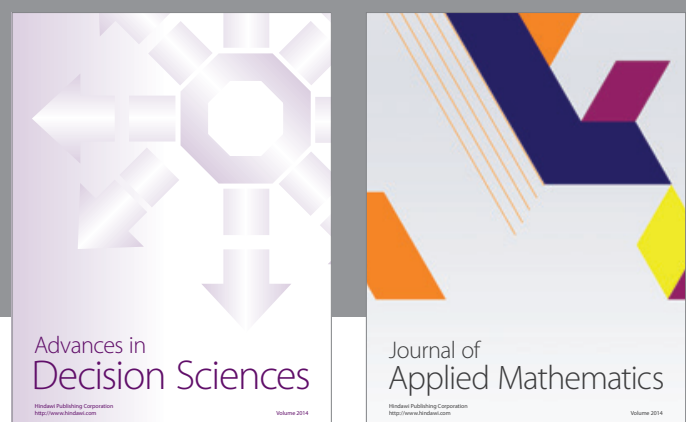

Journal of

Applied Mathematics
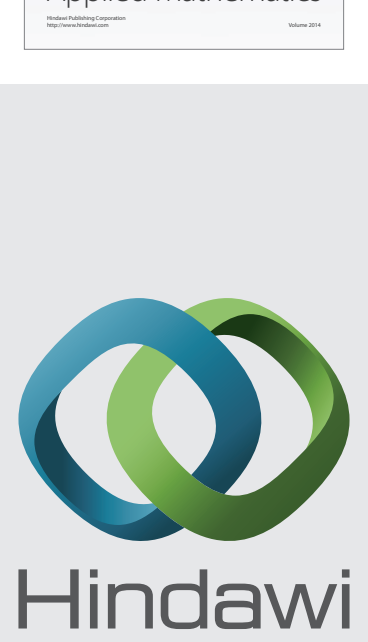

Submit your manuscripts at http://www.hindawi.com
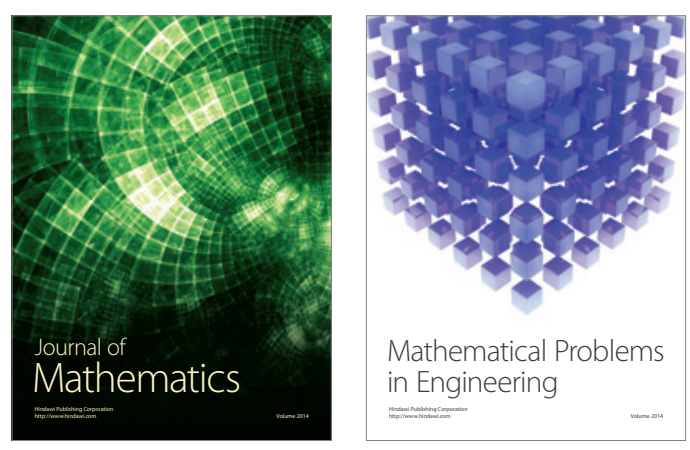

Mathematical Problems in Engineering
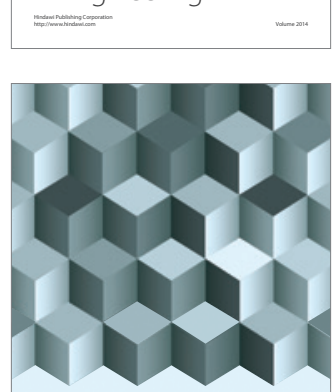

Journal of

Function Spaces
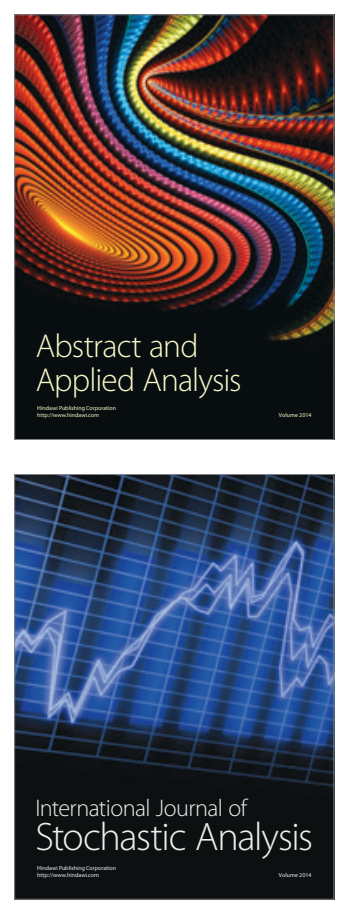

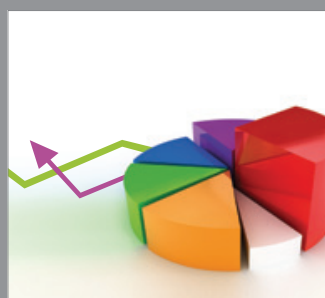

ournal of

Probability and Statistics

Promensencen
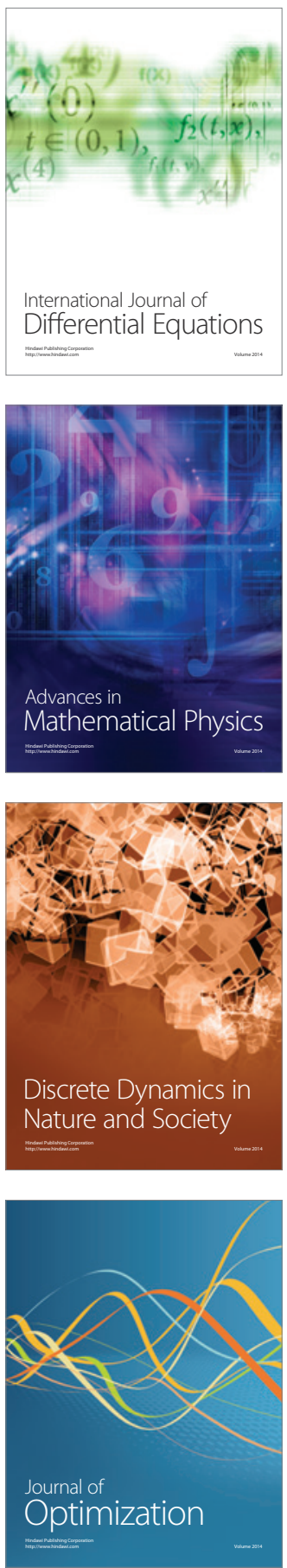